\title{
ACTITUD HACIA LA INVESTIGACIÓN EN LA FACULTAD DE CIENCIAS DE LA SALUD DE LA UNIVERSIDAD PRIVADA NORBERT WIENER, 2017
}

\author{
ATTITUDE TOWARDS RESEARCH IN THE FACULTY \\ OF SCIENCES OF HEALTH OF UNIVERSIDAD PRIVADA \\ NORBERT WIENER, 2017
}

CÉSAR ARELLANO-SACRAMENTO

Universidad Norbert Wiener

ROCÍO VICTORIA HERMOZA-MOQUILLAZA

Dirección General de Medicamentos, Insumos y Drogas

MARIO ELÍAS-PODESTÁ

Universidad Continental

MÁXIMO RAMÍREZ-JULCA

Universidad Alas Peruanas

\section{RESUMEN}

La investigación es base fundamental en el progreso de la ciencia, y la universidad es la institución que debe alentar a sus estudiantes a realizarla. El objetivo del presente estudio fue determinar la actitud hacia la investigación de los estudiantes de la Facultad de Ciencias de la Salud de la Universidad Privada Norbert Wiener (UPNW) en Lima, 2017. Es un estudio cuantitativo, observacional, transversal. Los sujetos fueron 1300 estudiantes de la Facultad de Ciencias de la Salud (excepto Medicina Humana), del V al X ciclo académico, hombres y mujeres, de entre 18 y 50 ańos, quienes accedieron a la ficha de recolección de datos a través de la plataforma virtual. El instrumento utilizado fue originalmente dirigido a alumnos de la carrera de odontología y en forma presencial; por ello, se realizó una validación de constructo en este nuevo grupo poblacional (estudiantes de la Facultad de Ciencias de la Salud), de lo que resultó un nuevo instrumento de 13 ítems con validez y confiabilidad comprobada por el estudio. Se procedió también a calcular un baremo. El 80,2 \% de los sujetos fueron mujeres. Se evidencia menos participación de estudiantes en tanto se acercan al último ciclo. La mayor cantidad de sujetos fueron de la carrera de Odontología (23,2 \%). Hubo semejanza en la frecuencia de rangos de edad "hasta 23 ańos" y "de 24 a 27" (26,1 \% y 27,2 \%, respectivamente). El 50,9\% de los estudiantes evidenciaron una actitud regular hacia la investigación.

Palabras clave: actitud, investigación, estudiantes del área de la salud, Perú.

\section{ABSTRACT}

Research is a fundamental component in the progress of science, with the university being the institution that should encourage its students to perform. The objective of the present study was to determine the attitude towards the research of the students 
of the Faculty of Health Sciences of the UPNW in Lima 2017. Quantitative, observational, cross-sectional study. The subjects were 1300 students of the Faculty of Health Sciences (except Human Medicine and Pharmacy and Biochemistry), from $\mathrm{V}$ to $\mathrm{X}$ academic cycle, of both sexes, between 18 to 50 years of age, who had available the collection form of data in its virtual platform. The instrument used was originally aimed at students of the stomatology career and in person, therefore, a construct validation was performed in this new population group (students of the Faculty of Health Sciences), resulting in a new instrument with 13 items with validity and reliability verified by the study. We also proceeded to calculate a scale. $80.2 \%$ of the subjects were women; as the cycles progressed ( $\mathrm{V}$ to $\mathrm{X}$ ), the number of participating subjects decreased. The largest number of subjects belonged to the career of Dentistry $(23,2 \%)$. There was similarity in the frequency of age ranges of "Up to 23 years" and "From 24 to 27 " (26,1 and 27,2\%). 50,9\% of the students had a regular attitude toward research. The majority of the students of the different health careers had a regular attitude toward research.

Key words: Attitude, Research, Students, Health Occupations, Peru.

\section{INTRODUCCIÓN}

Según $\mathrm{OCDE}^{1}$, estamos en un periodo de comercialización de la educación superior, el cual se caracteriza por utilizar mecanismos de enseñanza menos exigentes y rigurosos.

En el Perú, la Ley Universitaria, aprobada en 2014, está centrada en la calidad de la educación superior, y reafirma al Ministerio de Educación como ente rector de la política de aseguramiento de la calidad de la educación superior universitaria. Por medio de la Sunedu (Superintendencia Nacional de Educación Superior Universitaria) verifica el cumplimiento de las condiciones básicas de calidad tanto en universidades públicas como privadas; así mismo, fiscaliza si los recursos públicos han sido destinados a fines educativos ${ }^{1}$.

El Licenciamiento Institucional en el Perú enfatiza en la «existencia de políticas, normas y procedimientos para el fomento y realización de la investigación como una actividad esencial y obligatoria de la universidad $»^{2}$. Esto implica que la institución debe saber, entre otras cosas, qué es lo que piensa el estudiante universitario con respecto a la investigación.

La investigación es la acción y efecto de investigar ${ }^{3}$. Investigar es realizar actividades intelectuales y experimentales de modo sistemático, con el propósito de aumentar los conocimientos sobre una determinada materia ${ }^{4}$. Por un lado está la investigación formativa, una técnica didáctica para difundir la información existente sobre un tema y lograr que el estudiante, que busca ser formado profesionalmente y no en investigación, incorpore conocimientos sobre el tema tratado 5 .

También se considera la formación para la investigación que, según Guerrero, es el conjunto de acciones orientadas a favorecer la apropiación y desarrollo de habilidades, actitudes y conocimientos necesarios para que los participantes en la educación puedan desempeñar con éxito actividades productivas asociadas a la investigación científica y afines ${ }^{6}$. 
En este marco, y entendida la actitud como un estado neural de disposición a reaccionar a una determinada clase de objetos, no como son, sino como se conciben $^{7}$, definimos actitud hacia la investigación como la condición o estado caracterizado por sentimientos de interés del estudiante universitario con respecto a la investigación ${ }^{8}$.

Estudiantes de Odontología refieren sentirse confundidos con la elaboración de la pregunta de investigación, no por su redacción, sino porque usualmente estos cursos son llevados antes de iniciar sus prácticas clínicas (sienten que no saben nada de la carrera aún, por ello no enfocan un tema). Esta dificultad empeora cuando el tema lo elige la universidad. Otros problemas que manifiestan es el poco tiempo que los asesores les dan, su falta de preparación (deben confiar en ellos desde el inicio del proyecto), que subvaloren sus temas, la falta de estandarización de los asesores y el tiempo que le quita la investigación a la parte clínica, que es su prioridad ${ }^{9}$.

En otro contexto, docentes comentan que los estudiantes tienen dificultades para encontrar información científica (desconocen cómo localizar fuentes bibliográficas, seleccionar la información y evaluarla) ${ }^{10}$.

Una actitud favorable hacia la investigación permitiría al estudiante familiarizarse y alcanzar cierto dominio académico, propiciando que los problemas científicos sean abordados sistemáticamente, y ayudarlos a enfrentar y desarrollar con seguridad y crítica los retos y requisitos académicos, entre estos, la tesis universitaria ${ }^{11}$.

El objetivo del estudio fue determinar la actitud hacia la investigación de los estudiantes de la Facultad de Ciencias de la Salud de la UPNW en Lima en 2017.

\section{MATERIAL Y MÉTODO}

Estudio observacional, descriptivo, transversal. Se utilizó un instrumento (escala) validado $^{8}$ para medir la actitud hacia la investigación en estudiantes universitarios de carreras de Ciencias de la Salud. Ya que el instrumento original fue validado en un grupo poblacional diferente, se procedió a la validación con arreglo al grupo poblacional del presente estudio, utilizando análisis de componentes principales, análisis factorial (rotación Varimax) y la fiabilidad alfa de Cronbach (global y por factores encontrados). La encuesta fue colocada en la plataforma on line de la Universidad, accesible para el estudiante al ingresar con su usuario y contraseña.

Los criterios utilizados fueron: estudiantes de 18 a 50 años de edad, del V a X ciclo académico (los investigadores presuponen que estudiantes de ciclos menores no han recibido suficiente instrucción en cursos de investigación para medir una adecuada actitud).

Se realizaron las reuniones pertinentes con el área de Sistemas de la Universidad para la adaptación de la encuesta a la plataforma institucional. A fin de comprobar la operatividad del instrumento en su versión on line se lanzó previamente una prueba piloto en estudiantes del I al IV ciclo de la misma Facultad (estos resultados no entraron en la frecuencia global). La encuesta fue puesta a disposición de los alumnos entre junio y julio del periodo académico 2017-I (13-15. ${ }^{a}$ semana de clases). 
Las Escuelas encuestadas fueron: Enfermería, Tecnología Médica en Terapia Física y Rehabilitación, Odontología, Tecnología Médica en Laboratorio Clínico y Anatomía Patológica, Nutrición Humana, Psicología, Obstetricia; no fue incluida la carrera de Medicina Humana, que siendo nueva carrera no disponía de la administración de los últimos ciclos.

El instrumento original fue de 27 ítems sobre actitud (con 5 alternativas de respuesta). Se añadió una pregunta respecto al lugar donde llenó la encuesta. El área de Sistemas de la Universidad procesaría los datos de identificación como sexo, fecha de nacimiento, carrera, ciclo académico.

Según el instrumento original, a mayor valor, una mayor actitud, y a menor valor, menor actitud; se colocó un punto por cada respuesta (mínimo posible 27 y máximo posible 135). Los resultados del estudio fueron sometidos a un cálculo de confiabilidad global y por factores para asegurar la consistencia. Para el presente estudio se utilizó la escala Likert de cinco alternativas.

El proyecto fue revisado y aprobado en una primera instancia por un comité ad hoc conformado por el Rector, el Vicerrector, el Director de Desarrollo de la Investigación y el Director del Centro de Investigación; y, en una segunda instancia, fue revisado por el Comité Institucional de Ética de la Investigación de la UPNW.

La presente investigación se ciñó a las normas internacionales y nacionales sobre investigación en humanos. Se redactó y envió la documentación necesaria a todas las instancias y personas involucradas en el recojo de datos, y se siguió el procedimiento metodológico que mejor se adaptó a las circunstancias del estudio. Se salvaguardará los datos personales de los participantes. El estudio incluyó, al inicio de la encuesta, un texto que incluía el objetivo del estudio y la solicitud de consentimiento de modo virtual.

Se utilizó el programa estadístico SPSS v.22 para el análisis descriptivo e inferencial, y los programas Word y Excel, para los documentos y generación de las tablas y gráficos, respectivamente. La edad en años se dividió en cuartiles para la interpretación.

\section{RESULTADOS}

Fueron encuestados en total 1300 estudiantes del periodo 2017-I en la Facultad de Ciencias de la Salud quienes cumplieron los criterios de inclusión. La media de edad de los participantes fue de 27,96 años (DS de 6,24); el 80,2\% fueron mujeres. Mientras avanzaban los ciclos (V al X) disminuyó la cantidad de alumnos participantes. La mayoría fueron alumnos de Odontología (23,2\%). Respecto a las carreras, la mayoría respondió la encuesta "en una PC o laptop en casa" (45,8\%). Se obtuvo una semejanza en porcentajes en los rangos de edad de "hasta 23 años" y "de 24 a 27 ” (26,1 y 27,2 \%, respectivamente) (véase Tabla 1).

Se contrastó el número y constitución de los factores con el resultado del instrumento original, siguiendo la secuencia mencionada en materiales y métodos. Los cálculos fueron aceptables en todas las pruebas mencionadas, excepto cuando se calculó la fiabilidad en cada factor obtenido; resultó negativo o con consistencia interna baja, lo cual motivó eliminar 14 ítems. Los ítems que quedaron se mantuvieron en su posición original para 
TABLA 1

Datos sociodemográficos de los alumnos

\begin{tabular}{llll}
\hline & & \multicolumn{1}{c}{$\mathrm{n}$} & \multicolumn{1}{c}{$\%$} \\
\hline Sexo & Mujer & 1042 & 80,2 \\
Ciclo académico & Hombre & 258 & 19,8 \\
& 5 & 367 & 28,2 \\
& 6 & 272 & 20,9 \\
& 7 & 257 & 19,8 \\
& 8 & 232 & 17,8 \\
& 9 & 111 & 8,5 \\
& 10 & 61 & 4,7
\end{tabular}

Edad en categorías Hasta 23 años $339 \quad 26,1$

De 24 a $27 \quad 354 \quad 27,2$

De 28 a $31 \quad 302 \quad 23,2$

Mayor a $31 \quad 305 \quad 23,5$

\begin{tabular}{llll} 
Carreras & Enfermería & 269 & 20,7 \\
& TM Terapia Física & 293 & 22,5 \\
Odontología & 302 & 23,2 \\
TM Laboratorio Clínico & 187 & 14,4 \\
Nutrición Humana & 17 & 1,3 \\
Psicología & 100 & 7,7 \\
Obstetricia & 132 & 10,2 \\
Llenó la encuesta en: & & \\
Una PC o laptop en casa & 596 & 45,8 \\
La universidad (PC en clase) & 137 & 10,5 \\
La universidad (PC en aula libre) & 131 & 10,1 \\
Un teléfono inteligente (smartphone) & 215 & 16,5 \\
Una cabina de internet & 114 & 8,8 \\
Otro & 107 & 8,2 \\
\hline \multicolumn{2}{l}{ TOTAL } & 1300 & 100
\end{tabular}


ser analizados, es decir, con el número que presentaron en el instrumento original (véase Anexo).

Al analizar la reducción de dimensiones en base a la revisión de los componentes principales, con solo 13 de los 27 ítems del instrumento $(8,9,10,11$, $12,13,16,17,18,21,23,26,27)$ se encontró una reducción a dos factores que explican el 57,94 \% de la variabilidad total (Factor 1: 42,68 \% y Factor 2: $15,25 \%)$ de los datos; valores mayores a los encontrados en el instrumento original. La prueba de esfericidad de Bartlett y la medida Kaiser-Meyer-Olkin (KMO) confirmaron la adecuación del grupo de ítems para realizar el análisis factorial exploratorio.

El valor de KMO fue de 0,91 , por lo que la técnica de reducir información es adecuada. Además, en la esfericidad de Bartlett fue encontrado un valor $p$ significativo de 0,000 (las variables están correlacionadas o tienen una varianza común). El análisis factorial encontró que el Factor 1 (dominio: Acción) estuvo conformado por los ítems 9, 11, 17, 18, 21, 23, 26, 27, y el Factor 2 (dominio: Decisión), por los ítems $8,10,12,13$,
16. Asimismo, el cálculo de alfa de Cronbach global fue de 0,602 (lo cual es aceptable), siendo para el Factor 1 de 0,896 , y para el Factor 2 de 0,779.

El puntaje con los ítems resultantes se valoró en base a que, a un mayor valor, una mejor actitud; el mínimo posible de respuesta fue 13, y el máximo, 65. Para el cálculo del baremo fueron permutados al valor extremo los ítems negativos $(8,10$, $12,13,16$,$) . En base al resultado de la$ suma de ítems positivos y negativos permutados fue calculada la baremación del nuevo instrumento. Se utilizó la fórmula $\mathrm{X}_{+}-\left(\mathrm{DS}^{*} 0.75\right)$, donde $\mathrm{X}$ es la media aritmética y DS la desviación estándar, resultando Mala: de 13 a 40, Regular: de 41 a 48, Buena: de 49 a 65 .

Se encontró que el 50,9\% (662 de 1300) de los estudiantes de la Facultad de Ciencias de la Salud registraron un nivel Regular de actitud hacia la investigación (véase Tabla 2).

Las frecuencias más elevadas de actitud fueron en el nivel Regular en las carreras de Enfermería, Tecnología Médica en Terapia Física y Rehabilitación y Odontología $(11,5 \%, 11,0 \%, 11,2 \%$,

TABLA 2

Nivel de actitud hacia la investigación según Carrera

\begin{tabular}{lrrrrrrrr}
\hline \multicolumn{1}{c}{ CARRERA } & \multicolumn{1}{c}{ MALA } & \multicolumn{2}{c}{ REGULAR } & \multicolumn{2}{c}{ BUENA } & \multicolumn{2}{c}{ Total } \\
\hline & $\mathrm{n}$ & $\%$ & \multicolumn{1}{c}{$\mathrm{n}$} & $\%$ & $\mathrm{n}$ & $\%$ & $\mathrm{n}$ & $\%$ \\
Enfermería & 76 & 5,8 & 149 & 11,5 & 44 & 3,4 & 269 & 20,7 \\
TM Terapia Física & 86 & 6,6 & 143 & 11,0 & 64 & 4,9 & 293 & 22,5 \\
Odontología & 87 & 6,7 & 146 & 11,2 & 69 & 5,3 & 302 & 23,2 \\
TM Laboratorio Clínico & 38 & 2,9 & 89 & 6,8 & 60 & 4,6 & 187 & 14,4 \\
Nutrición Humana & 2 & 0,2 & 9 & 0,7 & 6 & 0,5 & 17 & 1,3 \\
Psicología & 30 & 2,3 & 50 & 3,8 & 20 & 1,5 & 100 & 7,7 \\
Obstetricia & 29 & 2,2 & 76 & 5,8 & 27 & 2,1 & 132 & 10,2 \\
\hline & 348 & 26,8 & 662 & 50,9 & 290 & 22,3 & 1300 & 100,0
\end{tabular}


respectivamente). Odontología tiene la mayor frecuencia de nivel de actitud Buena (5,3\%). Asimismo, también tiene la mayor frecuencia de Mala actitud $(6,7 \%)$, seguido de cerca por Tecnología Médica en Terapia Física y Rehabilitación (véase Tabla 2).

Cuando comparamos actitud con edad, observamos al inicio (hasta 23 años) una mayor frecuencia en el nivel Mala actitud, pero mientras la edad cambia a categorías de mayor edad estas se asemejan en frecuencia (véase Gráfico 1). Con respecto a la relación entre actitud y ciclo académico, el nivel Regular tiene una marcada tendencia a disminuir mientras cambian los ciclos hacia delante (del V al X). Lo mismo sucede con los niveles Bueno y Regular, pero no de forma tan marcada (véase Gráfico 2).

Los resultados individuales de los 13 ítems se muestran en el Gráfico 3.

\section{GRÁFICO 1}

Relación entre edad y nivel de actitud hacia la investigación de los alumnos de la Facultad de Ciencias de la Salud

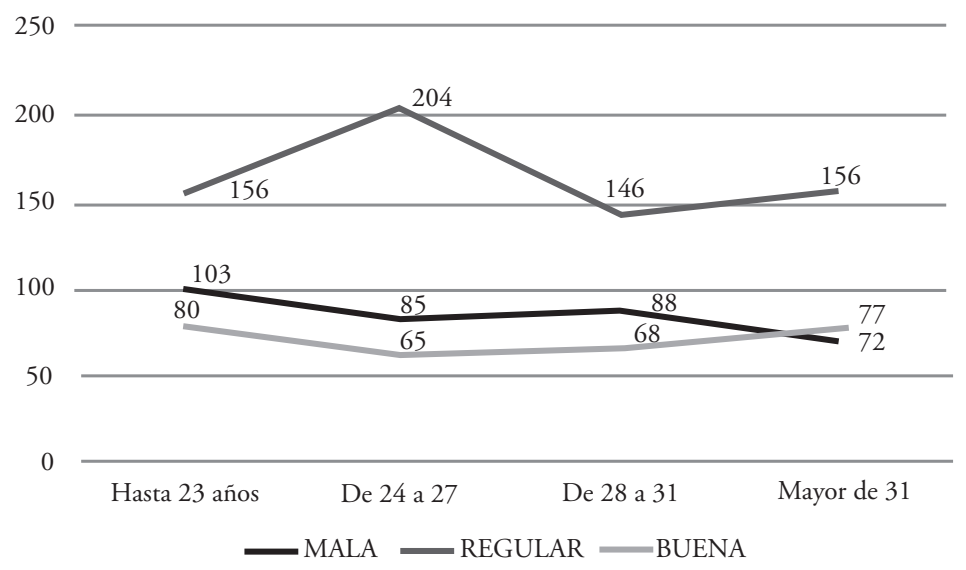

\section{GRÁFICO 2}

Relación entre ciclo académico y nivel de actitud hacia la investigación de los alumnos de la Facultad de Ciencias de la Salud

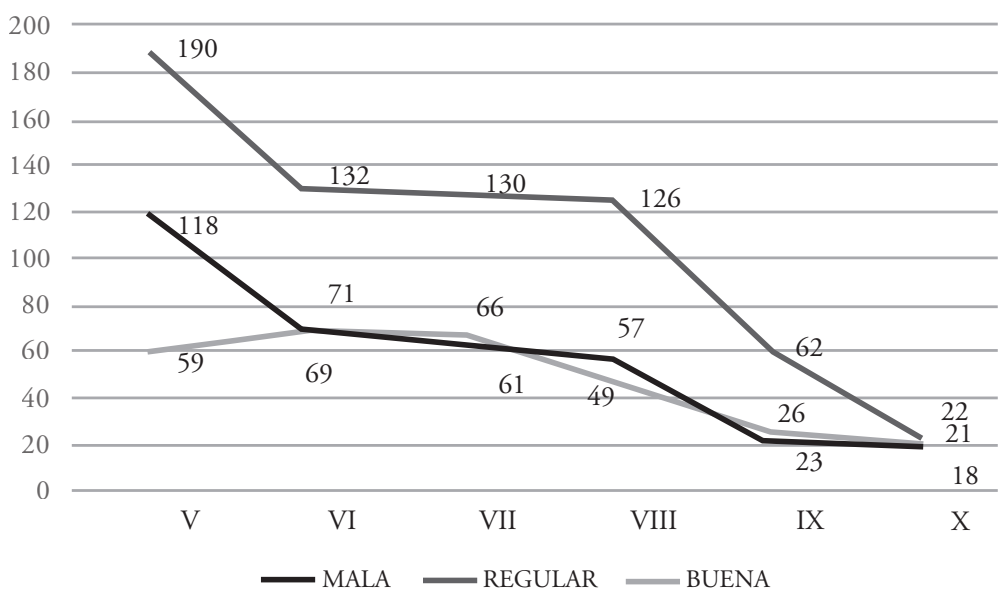




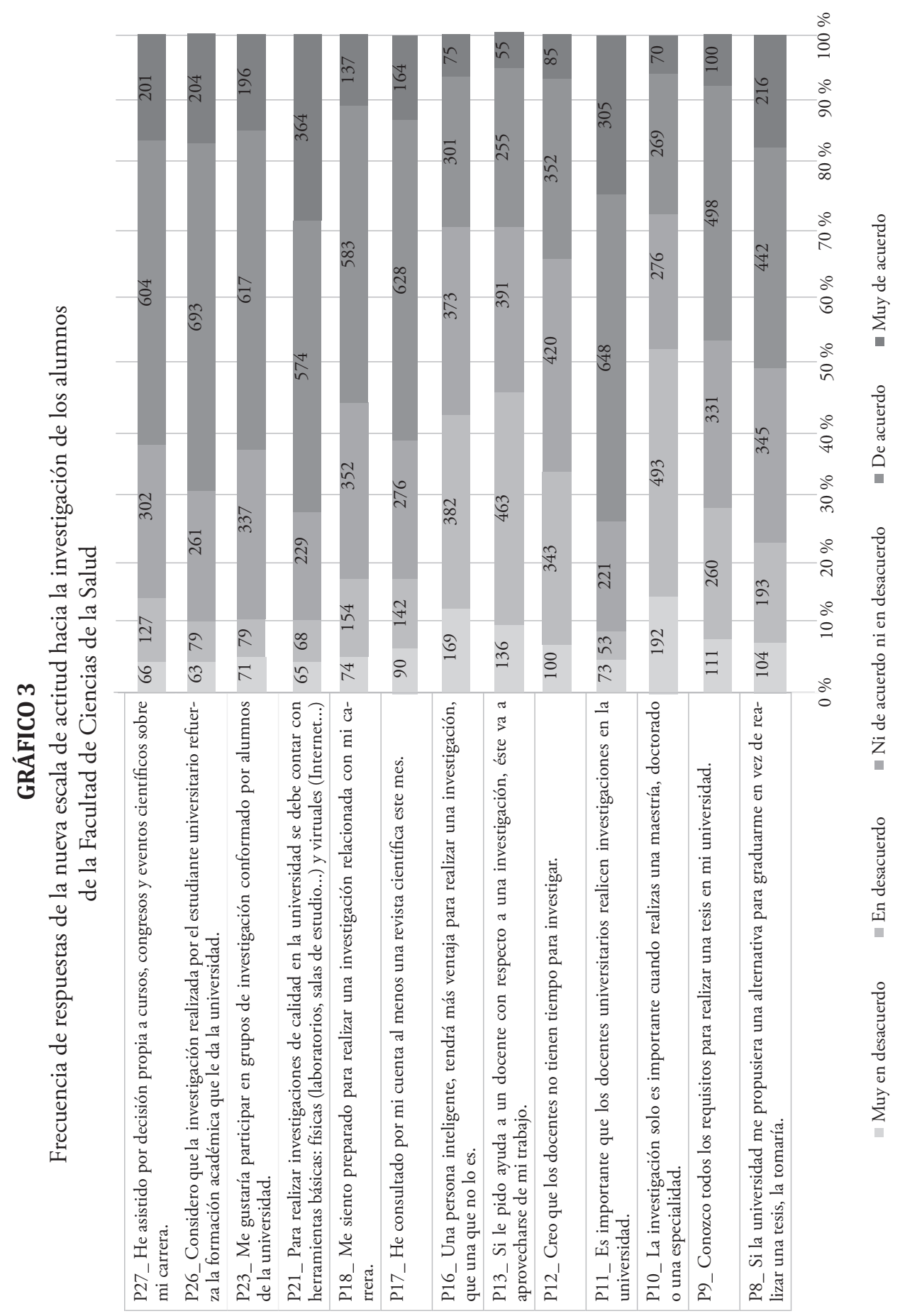




\section{DISCUSIÓN}

No se encontró otros estudios en los que interviniera toda una Facultad de Ciencias de la Salud ni que mencionen puntos de corte estrictos para hacer comparaciones directas; se realizarán comparaciones en caso de mencionar puntuaciones globales y de estudiantes de carreras individuales de salud. No se toma en cuenta estudios en carreras diferentes a ciencias de la salud. Si bien en el estudio no se considera estudiantes de Medicina, se realizarán comparaciones con el global de estudiantes en caso se crea necesario. Se incluirá la discusión de los ítems más relevantes. Se discutirá solo investigaciones hasta de siete años de antigüedad.

Según De la $\mathrm{Cruz}^{12}$, los estudiantes de Psicología de su estudio presentaron una actitud positiva hacia la formación científica de 3,18 puntos. Manifiesta una conformidad con el valor que presenta; sin embargo, no establece si el valor es aceptable (no menciona ni mínimo ni máximo para hacer una estimación). En el presente estudio se encontró que los estudiantes de Psicología presentaron $20 \%$ (20 de 100) de Buena actitud.

Según Jurado ${ }^{13}$, en estudiantes del último año de Enfermería se encontró resultados medianamente favorables en un $54 \%$ (54 de 100). Los resultados son similares a los del presente estudio, que encontró una mayor frecuencia en el nivel Regular, 55,39 \% (149 de 269), en los estudiantes de Enfermería.

Villamizar et al. ${ }^{14}$ encontraron una categoría alta en actitud hacia la investigación en 233 estudiantes de Psicología (media de 235,45). En el presente estudio se encontró valores menores, un $20 \%$ (20 de 100), en el nivel de actitud Buena en estudiantes de Psicología.
Mercado $^{15}$, en un estudio en el que participaron estudiantes de Medicina Humana, encontró un 78,72 \% (de un total de 94 estudiantes) con categoría Alto al hablar de actitud hacia la investigación. En el presente estudio, tomando en cuenta el resultado global, se encontró 22,3\% (290 de 1300) en nivel de actitud Buena.

En su estudio, Alonso et al. ${ }^{16}$, en 32 estudiantes de Enfermería encontraron una actitud positiva hacia la investigación de 53,2\%; fue, además, la mayor frecuencia encontrada. El presente estudio encontró un menor porcentaje en actitud Buena: 16,36 \% (44 de 269).

Chara y Olortegui ${ }^{17}$, en 100 estudiantes de Enfermería del primero al décimo ciclo, encontraron que más de la mitad de los estudiantes encuestados mostró una actitud medianamente favorable hacia la investigación. Los resultados son similares a los del presente estudio, que encontró 55,39 \% (149 de 269) en el nivel Regular.

Silva ${ }^{18}$, en estudiantes de Medicina, encontró una media de actitud de 2,98 (DE:0,59), siendo caracterizado como Regular, aunque el valor de indiferencia del estudiante sería de 3. En el presente estudio, el 50,9 \% del global de estudiantes obtuvo un nivel Regular $(50,9 \%, 662$ de 1300$)$.

Con respecto al ítem 8 ("Si la universidad me propusiera una alternativa para graduarme en vez de realizar una tesis, la tomaría”) la mayoría de estudiantes, $34 \%$ del total de encuestados, marcó De acuerdo. Esto se presta a analizar las razones. Una de ellas, suponemos, sería la burocratización de la tesis. Esto ameritaría una revisión profunda sobre los lineamientos 
y protocolos utilizados en cada escuela profesional, para buscar unificación de criterios entre docentes de metodología, asesores, jurados, comité de revisión de proyectos, dirección de escuela (entre otros entes involucrados); así, ninguno de los actores mencionados truncaría el avance de la investigación porque tiene una "visión" diferente del resto. Esto agilizaría los tiempos de la presentación de proyecto, ejecución, revisión y sustentación de la tesis como producto. Además, la Universidad podría analizar y apoyar económicamente los mejores proyectos de tesis. Sería necesario evaluar la posibilidad de realizar concursos de fondo (económico) de investigación para estudiantes. La evidencia ${ }^{19}$ nos dice que, si bien no es conocido el impacto de esta modalidad, tanto en docentes como alumnos, se ha incrementado el número de publicaciones en las instituciones que lo han implementado.

Con respecto al ítem 11 ("Es importante que los docentes universitarios realicen investigaciones en la universidad"), los estudiantes consideran que el docente universitario debe ser el primero en realizar investigación; 648 de 1300 están De acuerdo. Sin embargo, para lograr que los docentes realicen investigación la Universidad debería también motivarlos, por ejemplo, publicar con filiación institucional, otorgar becas y puntos para futuras contrataciones, etc.

El 47,5\% del total de encuestados respondió estar De acuerdo con el ítem 23 ("Me gustaría participar en grupos de investigación conformado por alumnos de la Universidad"). Este bajo porcentaje podría deberse a que no saben qué implica o si será una actividad extra con respecto a los cursos normales del currículo. Estos grupos deben ser creados como iniciativa de la Universidad y encargarlos a los docentes que dictan los cursos de investigación, ya que estos deben plantear el camino para el inicio de las actividades; además, se debe motivar al estudiante (y de alguna manera al docente) con incentivos de becas, puntos extra en los cursos, etc.

El análisis y seguimiento de estos resultados llevará a la mejora de la calidad del servicio académico con respecto a la formación para la investigación de los estudiantes de la Facultad de Ciencias de la Salud de la Universidad Privada Norbert Wiener.

Se recomienda continuar constantemente la medición de la actitud hacia la investigación en los estudiantes de Ciencias de la Salud, sumando a aquellas carreras que no fueron tomadas en cuenta en el presente estudio, e incluyéndolas en futuras validaciones.

\section{CONCLUSIÓN}

La mayoría de los estudiantes de las distintas carreras de salud mostraron una actitud Regular hacia la investigación. De entre todas las carreras incluidas, Enfermería, Tecnología Médica en Terapia Física y Rehabilitación, y Odontología mostraron una mayor frecuencia en actitud Regular y fueron de similar proporción. 


\section{ANEXO}

\begin{tabular}{|c|c|c|c|c|c|}
\hline \multicolumn{6}{|c|}{$\begin{array}{l}\text { Instrumento para medir la actitud hacia la investigación en estudiantes de pregrado } \\
\text { de Ciencias de la Salud }\end{array}$} \\
\hline \multicolumn{6}{|c|}{ Marca con una equis (X) la alternativa de respuesta que creas conveniente (solo uno por ítem). } \\
\hline \multicolumn{6}{|c|}{$\begin{array}{c}\text { Recuerda que NO HAY RESPUESTAS CORRECTAS NI INCORRECTAS. } \\
\text { Asegúrate de contestar todos los ítems. }\end{array}$} \\
\hline & $\begin{array}{c}\text { Muy en } \\
\text { desacuerdo }\end{array}$ & $\begin{array}{c}\text { En } \\
\text { desacuerdo }\end{array}$ & $\begin{array}{l}\text { Ni de acuerdo } \\
\text { ni en desacuerdo }\end{array}$ & $\begin{array}{c}\text { De } \\
\text { acuerdo }\end{array}$ & $\begin{array}{l}\text { Muy de } \\
\text { acuerdo }\end{array}$ \\
\hline \multicolumn{6}{|l|}{$\begin{array}{l}\text { Si la universidad me propusiera una alternativa } \\
\text { para graduarme en vez de realizar una tesis, la to- } \\
\text { maría. }\end{array}$} \\
\hline \multicolumn{6}{|l|}{$\begin{array}{l}\text { Conozco todos los requisitos para realizar una tesis } \\
\text { en mi universidad. }\end{array}$} \\
\hline \multicolumn{6}{|l|}{$\begin{array}{l}\text { La investigación solo es importante cuando realizas } \\
\text { una maestría, doctorado o una especialidad. }\end{array}$} \\
\hline \multicolumn{6}{|l|}{$\begin{array}{l}\text { Es importante que los docentes universitarios reali- } \\
\text { cen investigaciones en la universidad. }\end{array}$} \\
\hline \multicolumn{6}{|l|}{$\begin{array}{l}\text { Creo que los docentes no tienen tiempo para in- } \\
\text { vestigar. }\end{array}$} \\
\hline \multicolumn{6}{|l|}{$\begin{array}{l}\text { Si le pido ayuda a un docente con respecto a una } \\
\text { investigación, éste va a aprovecharse de mi trabajo. }\end{array}$} \\
\hline \multicolumn{6}{|l|}{$\begin{array}{l}\text { Una persona inteligente, tendrá más ventaja para } \\
\text { realizar una investigación, que una que no lo es. }\end{array}$} \\
\hline \multicolumn{6}{|l|}{$\begin{array}{l}\text { He consultado por mi cuenta al menos una revista } \\
\text { científica este mes. }\end{array}$} \\
\hline \multicolumn{6}{|l|}{$\begin{array}{l}\text { Me siento preparado para realizar una investiga- } \\
\text { ción relacionada con mi carrera. }\end{array}$} \\
\hline \multicolumn{6}{|l|}{$\begin{array}{l}\text { Para realizar investigaciones de calidad en la uni- } \\
\text { versidad se debe contar con herramientas básicas: } \\
\text { físicas (como laboratorios, salas de estudio, libros } \\
\text { actualizados) y virtuales (como Internet, bases de } \\
\text { datos). }\end{array}$} \\
\hline \multicolumn{6}{|l|}{$\begin{array}{l}\text { Me gustaría participar en grupos de investigación } \\
\text { conformado por alumnos de la universidad. }\end{array}$} \\
\hline \multicolumn{6}{|l|}{$\begin{array}{l}\text { Considero que la investigación realizada por el es- } \\
\text { tudiante universitario refuerza la formación acadé- } \\
\text { mica que le da la universidad. }\end{array}$} \\
\hline $\begin{array}{l}\text { He asistido por decisión propia a cursos, congresos } \\
\text { y eventos científicos sobre mi carrera. }\end{array}$ & & & & & \\
\hline
\end{tabular}




\section{REFERENCIAS BIBLIOGRÁFICAS}

1. OCDE. Avanzando hacia una mejor educación para Perú. Lima: OCDE; 2016.

2. Sunedu. El Modelo de Licenciamiento y su Implementación en el Sistema Universitario Peruano». Lima: SUNEDU; 2016.

3. Real Academia Española. Diccionario de la lengua española, $22^{\circ}$ Edición. Consulta: 20 de octubre 2017. Disponible en: <http://lema.rae.es/drae/?val=investigación $>$.

4. Real Academia Española. Diccionario de la lengua española, $22^{\circ}$ Edición. Consulta: 20 de octubre 2017. Disponible en: <http://lema.rae.es/drae/?val=investigar $>$.

5. Parra C. Apuntes sobre la investigación formativa. Educación y educadores. 2004; 7: 57-77. Consulta: 22 de octubre 2017. Disponible en: <http:// dialnet.unirioja.es/servlet/articulo?codigo $=2041050>$.

6. Miyahira J. La investigación formativa y la formación para la investigación en el pregrado [editorial]. Rev Med Hered. 2009; 20(3):119-122.

7. Liñan CGB. Psicometría. Lima: Fondo Editorial de la Universidad Inca Garcilaso de la Vega; 2009.

8. Arellano-Sacramento C, Hermoza-Moquillaza RV, Elías-Podestá M, Ramírez-Julca M. Actitud hacia la investigación de estudiantes universitarios en Lima, Perú. FEM 2017; 20 (4): 191-197.

9. Franco-Cortés AM. La investigación formativa en la Facultad de Odontología de la Universidad de Antioquia: vivencias de los estudiantes de pregrado. Rev Nac de Odont. 2015; $11(21)$.

10. Landu BJ, Dos Santos OM, João SJ, Mangovo MP, Tekuebua MJ, Tibúrcio WA. Diagnóstico de la situación de la actividad científica estudiantil en la Facultad de Medicina de Cabinda,
República Popular de Angola. MEDICIEGO 2012; 18 (1).

11. Mamani BOJ. Actitud hacia la investigación y su importancia en la elección de la modalidad de tesis para optar el título profesional. Revista Científica de Ciencias de la Salud. 201 1; 4(4):22-27.

12. De la Cruz VC. Actitudes hacia la investigación científica en estudiantes universitarios: Análisis en dos universidades nacionales de Lima. Revista Psique Mag. 2013; 2(1): 1-16.

13. Jurado VSC. Actitudes hacia la investigación en estudiantes de enfermería de la UNMSM, 2016 [Tesis]. Lima:UNMSM; 2017.

14. Villamizar G, Núñez K, Rolón J. Actitudes de los estudiantes de Psicología frente a la investigación. I+D Revista de Investigaciones. 2016; 7(1): 49-60.

15. Mercado RMR. Actitudes hacia la investigación en los estudiantes de la carrera de Medicina Humana de la Universidad Peruana Los Andes. Educ Med. 2018.

16. Alonso TJ, Alonso RA, Valadez DD. Actitud hacia la investigación científica de estudiantes de Enfermería. CuidArte. 2015; 4(7):22-35.

17. Chara P, Olortegui A. Factores asociados a la actitud hacia la investigación en estudiantes universitarios de Enfermería. CASUS. 2018;3(2):83-88.

18. Silva S, Zúñiqa-Cisneros J, Ortega-Loubon C, Yau A, Castro F, Barría-Castro JM. et al. Conocimientos y actitudes acerca de la investigación científica en los estudiantes de Medicina de la Universidad de Panamá. Archivos de Medicina. 2013; 9(3):1-10.

19. Nieto-Gutierrez W, Fernández-Chinguel JE, Taype-Rondan A, Pacheco-Mendoza J, Mayta-Tristán P. Incentivos por publicación científica en universidades peruanas que cuentan con escuelas de Medicina, 2017. Rev Peru Med Exp Salud Publica. 2018;35(2):354-6. doi: 10.17843/rpmesp.2018.352.3327. 